Running header: Re-envisioning depression treatment

\title{
Wider, faster, more: Re-envisioning the treatment and research of depression to address its public health burden in the United States
}

\author{
Lorenzo Lorenzo-Luaces, $\mathrm{PhD}^{1 *}$
}

${ }^{1}$ Department of Psychological and Brain Sciences, Indiana University, Bloomington, IN, USA

* correspondence regarding this article should be made to Prof. LL-L: $\underline{\text { lolorenz@iu.edu }}$

\footnotetext{
Acknowledgments: This work was supported in part by grant numbers KL2TR002530 and UL1TR002529 (A. Shekhar, PI) from the National Institutes of Health, National Center for Advancing Translational Sciences, Clinical and Translational Sciences Award (PI: LL-L). The funders had no role in the writing or drafting of the manuscript.

Conflict of interest: Prof. Lorenzo-Luaces has received consulting fees from Happify Health Inc, who had no role in the current work.
} 


\begin{abstract}
Depression and other internalizing disorder symptoms are leading causes of disability in the United States. Although there are effective interventions, the burden of disability attributable to internalizing disorder symptoms is not decreasing. I review clinical and epidemiological data to identify solutions to the public health burden of internalizing disorder symptoms. Contemporary research efforts have a focus on treatment development and the identification of biomarkers of response. However, these solutions are not scalable because internalizing disorders are much more common than usually appreciated and there are substantial disparities in access to mental health care. Improving the dissemination of individual interventions may also be of limited value given data suggesting that high rates of symptom remission can only be achieved with multiple (e.g., 4-10) treatment steps. The logic of stepped care approaches for internalizing disorders is well-supported by these data, but initial treatments likely should be low-intensity and "stepping up" should occur more quickly than usually done in most trials. Efforts that promise to make an impact in the public health burden of internalizing disorder symptoms need to consider their high prevalence and heterogeneity in the level of care as well as in the mechanisms that treatments may need to engage.
\end{abstract}

Keywords: Depression, anxiety, public health, disparities, global health, cognitive-behavioral therapy 
Depression is among the top 10 leading causes of disability in the United States and worldwide (Whiteford et al., 2013). Effective interventions for depression exist including various psychological treatments, the most studied of which are cognitive-behavioral therapies (CBTs; Lorenzo-Luaces, Lemmens, et al., 2021), and pharmacological interventions like selective serotonin reuptake inhibitors (SSRIs; Cipriani et al., 2018). Despite the existence of these treatments, the prevalence of depression does not appear to be decreasing, even in places where treatment accessibility has increased (Jorm et al., 2017; Ormel et al., 2021).

This article is a call to reform the existing treatment efforts for depression in the United

States, including treatment research. I review epidemiological and clinical which support the need to push for a broader array of treatments considered within studies, with larger and more representative samples, with faster switching between treatment modalities, and balancing pragmatic and scalable approaches with personalized efforts. The article ends with recommendations for future work. Most of the article is supported by studies that used the Diagnostic and Statistical Manual of Mental Disorder's (DSM) definition of major depressive disorder (MDD) but I reconcile the binary DSM-MDD classification with more dimensional classification approaches, including "transdiagnostic" ones (Kotov et al., 2017).

Society and the diagnosis and treatment of depression. In the United States (U.S.), $20.6 \%$ of individuals recall meeting the criteria for MDD at some point in their lives (Hasin et al., 2018). A very consistent finding in epidemiological studies is that stressors are associated with the onset of MDD (Hammen, 2005). Another consistent finding is that cisgender women (26.1\%) are more likely to meet the criteria for MDD when compared to cisgender men $(14.7 \%$; Hasin et al., 2018). Transgender men, transgender women, and gender non-conforming individuals are at elevated risks for depression compared to their cisgender counterparts (Pellicane \& Ciesla, 2021) and lesbian, gay, and bisexual individuals are also at elevated risk compared to their heterosexual counterparts (Newcomb \& Mustanski, 2010).

Race and ethnicity appear to have complex relations with depression in that high rates of 
depression are found in Non-Hispanic Whites (23.1\%) with higher rates being found among Native Americans (28.2\%). However, other groups that are minoritized, and more likely to experience psychosocial stressors (Pamplin II \& Bates, 2021), like Non-Hispanic Black (15.2\%) and Hispanic (16.2\%) adults may actually be at lower risk for lifetime depression compared to their Non-Hispanic White counterparts. Nonetheless, when they become depressed, racial and ethnic minorities can have more difficulty accessing treatment for mental health care (Alegría et al., 2008; Mojtabai, 2009). The prevalence of depression does not show large variations by geographic areas, though the presence of trained mental health providers is concentrated in large metropolitan areas (Kazdin \& Blase, 2011). Unsurprisingly, around $40 \%$ of people with MDD in the U.S. are untreated for their depression and only $20 \%$ or so receive care that is consistent with clinical guidelines (Kessler et al., 2003).

Most individuals do not experience remission on their first treatment. Accessing and completing a single treatment for depression is, however, little guarantee that an individual will experience symptom remission. In the World Health Organization World Mental Health Surveys ( $=80,332$; Harris et al., 2020), only 30.6\% of individuals reported finding that their initial treatment for depression was "helpful." While the probability that an individual will report that a treatment is helpful nearly doubles after the second treatment (58.2\%), each failed treatment is associated with a $15-25 \%$ risk of treatment dropout. After 10 treatment attempts, the conditional probability of individuals finding treatments effective approximates $100 \%$. However, few people who may benefit from that degree of treatment persist after so many treatment attempts $(21.5 \%)$, and it is unclear what predicts persistence in depression treatment (Harris et al., 2020).

Randomized controlled trials (RCTs) also support the idea that, on average, multiple treatment steps are required to achieve remission from depression. The Sequenced Treatment Alternatives to Relieve Depression $\left(\mathrm{STAR}^{*} \mathrm{D}\right)$ study $(\mathrm{N}=4,041)$ was a large and diverse sample of depressed outpatients. STAR*D began with an 8-week open trial of an SSRI with the option for an additional 3-4 treatment steps in which individuals were randomized to various pharmacological interventions, or CBT, if they had not experienced symptom remission. After the 
first treatment level, only $33 \%$ of patients had achieved symptom remission (Rush et al., 2006). After the final level, however, the overall remission rate was between $67-71 \%{ }^{1}$.

These studies suggest that high rates of remission in depression can be achieved but they can only be achieved by allowing individuals to access multiple (i.e., 5-10) treatment options. Given the low access to depression treatment in general, and the broad disparities in access to care, these data suggest a need to radically rethink treatment for depression. Most RCTs of psychological and pharmacological interventions, which are primarily focused on comparing one treatment to another or a treatment to a control control are ill-suited to treat a majority of patients to remission. The treatment of depression to remission is a dynamic process and should be studied as such.

Waiting for treatment development. It is possible that scientific discovery could produce a treatment for depression that is more efficacious than all other treatments in the way metfermin is a first-line treatment for diabetes (Nemeroff, 2021) or how antibiotics can treat most cases of MALT lymphoma associated with Helicobacter pylori infection (Filip et al., 2018). However, the past 40 years or so of research in the treatment of depression are replete with examples of interventions that purported to be superior to existing treatments only to be found roughly equally efficacious (Cuijpers, 2016) after research that is more rigorous, and less prone to biases, than the initial studies (Cuijpers \& Cristea, 2016; Driessen et al., 2010; Turner, 2013). While these patterns in the literature should not discourage treatment development research, there is a great need to devote resources to the study of optimal use of existing treatment resources, for example investigating the efficacy and cost-effectiveness of different sequencing strategies for existing interventions. Importantly, there is a large lag between the discovery of an innovative treatment and its implementation in clinical practice (e.g., the oft-cited 17year lag between discovery and innovation; Morris et al., 2011). Thus, it is unlikely that new treatments will make immediate impacts at a large scale and in a way that could that address disparities in access to treatments for depression.

Depression is more prevalent than usually thought. Somewhat surprisingly, and

\footnotetext{
${ }^{1}$ The rate reported varied across publications (see Pigott, 2011, 2015)
} 
differentiating depression from other causes of disability like diabetes or bipolar I (Takayanagi et al., 2014), many individuals forget they experienced episodes of depression, even those that met the DSM criteria (Wells \& Horwood, 2004). In studies in which individuals have been assessed repeatedly over their life course, the prevalence of MDD has ranged from 30-50\% by age 30 (Lorenzo-Luaces, 2015). This finding has been reported in samples recruited from New Zealand, (Moffitt et al., 2010; Wells \& Horwood, 2004), Australia (Wilhelm et al., 2006), Switzerland (Angst et al., 2016), and the United States (Rohde et al., 2013). High prevalence rates of MDD make sense if one conceptualizes depression as being on a continuum with "normal" sadness (Ruscio, 2019). However, the fact that many individuals have at least one point in their life when they experience such high levels of depressive symptoms suggests that, at a societal level, the treatment of individuals with depression symptoms needs to be scalable (i.e., something that 30-50\% of people could access). There are not, and may never be, enough specialty providers to dispense specialized psychological and pharmacological interventions to everyone who becomes depressed (Kazdin \& Blase, 2011). Proposals to expand the range of available providers (e.g., by increasing access to telehealth) are important (Ashwood et al., 2017). However, these proposals are still limited by the ratio of providers to patients (Kazdin \& Blase, 2011). More importantly, there are strong reasons to believe that not all individuals require such intensive, expensive, and difficult to access treatments.

Naturalistic recovery. While treatment-seeking individuals tend to have more chronic and relapsing courses than individual in outpatient and inpatient samples (Lorenzo-Luaces, 2015), for many individuals depression is a time-limited problem. In naturalistic samples, roughly $50 \%$ of individuals who recover do not go on to have another depressive episode in the 20-30 year follow-ups available (Eaton et al., 2008; Mattisson et al., 2007; Steinert et al., 2014). Moreover, epidemiological studies of first-onset depression suggest that around two-thirds of individuals recover from their depression 3-6 months after its onset (Eaton et al., 1997; Eaton et al., 2008; Spijker et al., 2002). Thus, depression is not a universally chronic and recurrent phenomenon (Lorenzo-Luaces, 2015; Monroe \& Harkness, 2011; Rottenberg et al., 2018). 
the field is the study of self-help materials, including smartphone applications (i.e., "apps"), websites, books, and group education (Cuijpers et al., 2019). Self-help can be provided with guidance by a professional or paraprofessional, known as guided self-help, or without such guidance, "unguided." Self-help comports with the preferences of most depressed individuals who because a substantial portion (28-44\%) report thinking that they do not need treatment (Mojtabai, 2009; Mojtabai et al., 2011) and most individuals (72-77\%) want to manage their depression "by themselves." CBTs are the family of psychological interventions that have been studied most often in self-help format though there also are self-help forms of psychodynamic (Lindegaard et al., 2020) and interpersonal (Bright et al., 2019) psychotherapies. Guided and unguided self-help CBT both appear more efficacious than inactive control conditions including waiting lists and care as usual (Cuijpers et al., 2019). In the handful of trials that have compared guided self-help CBT to individual face-to-face CBT, the treatment differences appear small and not statistically significant $(\mathrm{SMD}=-0.12,95 \%$ : $-0.54-0.31$; Cuijpers et al., 2019), although the likelihood of treatment dropout tends to be higher in guided self-help than in individual CBT $(\mathrm{OR}=1.44,95 \% \mathrm{CI}: 1.09$ - 1.89). Supporting its scalability, the guidance in guided self-help CBT can be relatively minimal. For example, my colleagues and I recently (LorenzoLuaces, Howard, et al., 2021) conducted a nationwide open trial $(\mathrm{N}=107)$ of self-help CBT in which guidance was provided by graduate and undergraduate students by way of a 30-minute videoconferencing or phone call session followed by 2-5 15-minute sessions over a 6 -week period. In other studies, guidance is delivery via weekly text messages or weekly e-mails and the format of the guidance does not appear to be a predictor of outcomes (see Cuijpers et al., 2010).

Investigators who study self-help approaches are often careful to describe how these approaches are not meant to entirely replace existing models of care (Schleider \& Weisz, 2017) but instead propose them as initial steps in stepped care programs. A stepped care program is one in which an individual starts a low-intensity treatment, and "steps up" to a higher intensity treatment after failing to experience symptom remission (Unützer et al., 2002). For example, the "Improving Mood Promoting Access to Collaborative Care Treatment" (IMPACT) model is a stepped care approach to the treatment of geriatric depression in which a nurse specialist 
facilitates the management of pharmacotherapy or brief CBT, followed by options to augment or switch treatments.

Some patients require intensive treatments. Despite the promise of low-intensity treatments, treatment programs must have relatively intensive treatments available. Adding psychotherapy or another medication (i.e., "augmenting") to an initial treatment or switching treatments both appear effective (Carvalho et al., 2014; van Bronswijk et al., 2019), and were steps in STAR*D. However, the observed rate of non-remission after 4-5 pharmacological/psychological treatments was still around 30\% (Pigott, 2011, 2015). Other relatively more intensive and expensive therapies, not available in the $\mathrm{STAR}^{*} \mathrm{D}$ trial, include electroconvulsive therapy (The UK ECT Review Group, 2003), deep brain stimulation (Kisely et al., 2018; Morishita et al., 2014), and ketamine (Bahji et al., 2021). They have shown varying levels of efficacy in treating depression though none show remission rates near $100 \%$. Thus, the need for low-intensity treatments needs to be balanced with the availability of interventions that are higher-intensity than existing psychological and pharmacological interventions.

Heterogeneity in prognosis and stratified care. The findings that the prognosis of depression is highly variable presents an opportunity to study "matching" individuals to the level of care that may be most appropriate for them (Lorenzo-Luaces et al., 2017; LorenzoLuaces et al., 2020). For example, in a Dutch sample of depressed and anxious outpatients $(\mathrm{N}=702)$, van Straten et al (2006) reported that there were no differences in short or long-term outcomes depression between a non-specific therapy intervention, brief therapy (BT), care as usual (CAU), and CBT. In the depressed patients in this sample $(\mathrm{N}=621)$, my colleagues and I developed a machine-learning algorithm predicting response from baseline symptom severity, unemployment, insomnia, the personality traits extraversion, and hostility. Most individuals (75\%) benefited as much from CBT, as from CAU, and BT. The other $25 \%$ of individuals $(\mathrm{n}=155)$ had higher remission rates in CBT $(60 \%)$ than in BT $(44 \%)$ or TAU (39\%; see also Lorenzo-Luaces et al., 2020). Recently, Delgadillo et al. (2021) showed how machine-learning algorithms like these could be used prospectively. They developed an algorithm of response to low vs. high intensity psychological intervention, which included symptom severity, unemploy- 


\footnotetext{
${ }^{2} \mathrm{~A}$ notable exception is biomarkers for medical conditions that have been implicated in depressive symptoms. For example, hypothyroidism is a common cause of depressive symptoms and low thyroid levels are pathognomonic of hypothyroidism.
}

ment, personality, and other variables, using observational psychotherapy data. In a cluster RCT in 951 patients, they used that algorithm to guide stepped care decisions (i.e., stratified care) and found that this approach produced better outcomes than stepped care without using information from the machine-learning algorithm (OR: 1.33, 95\% CI: 1.01-1.75).

Heterogeneity and treatment matching. In addition to being heterogeneous in prognosis, depression is heterogeneous in its presenting and co-morbid symptoms (Fried et al., 2020; Lorenzo-Luaces, Buss, et al., 2021). Thus, even focusing on interventions of the same "intensity" (e.g., outpatient psychotherapy), there is an opportunity to match individuals to mechanistically-different interventions that may be most effective for them. Using data-driven approaches, my colleagues and I have found that sociodemographic, contextual, and symptom features appear to predict differential response to CBT for depression vs.: antidepressants (DeRubeis et al., 2014), interpersonal psychotherapy (van Bronswijk et al., 2021), and positive psychotherapy (Lopez-Gomez et al., 2019), although these interventions are equally efficacious in comparative RCTs. Having adequate power to develop machine-learning algorithms to predict response to different interventions likely requires very large samples (e.g, Luedtke et al., 2019 recommended sample sizes 600-1000). Most studies that have attempted to use machinelearning approaches to predict outcomes have samples that are substantially smaller than these benchmark recommendations (Lorenzo-Luaces, Peipert, et al., 2021), so there is a need for large studies that could inform not just clinical staging but other forms of personalized treatment allocation.

Promise of biomarkers. There is great interest in supplementing self-report with biomarkers (Cuthbert \& Kozak, 2013) to identify more homogeneous subgroups of patients (Kennis et al., 2020) and to identify individuals who may differentially respond to existing interventions (Simon \& Perlis, 2010). Currently, no biological measure seems to be a replicable predictor of treatment outcomes (Harris et al., 2020) ${ }^{2}$, though there are promising candidates like baseline insula activity as a predictor of response to CBT vs. antidepressants (Dunlop et al., 2015; 
McGrath et al., 2013). Biomarker research appropriately continues on the promise that such biomarkers may be found. However, a comparison between depression and another medical complaint, upper respiratory symptoms, raises questions about whether biomarkers would be relevant to the treatment of most depessed patients. Prior to the COVID-19 pandemic, when an individual presented to a primary care setting with acute upper respiratory symptoms, they were not administered costly assessments (e.g., X-rays of the lungs) nor were they immediately given intensive treatments. Instead, medical providers usually assumed the symptoms were attributable to their most common cause: the common cold. Individuals were typically left to self-manage symptoms with relatively inexpensive and low burdensome treatments (e.g., over-the-counter medication), and prompted to return if symptoms remain beyond the natural course of the common cold. Even when symptoms persisted, individuals were not given expensive assessments but were instead treated with medications (e.g., antibiotics) intended to treat other common causes of upper respiratory symptoms (e.g., sinus infections). If biomarkers of response are found, it is likely that their role in clinical staging will be limited to subsets of patients with more treatment-resistant courses (i.e., those unlikely to remit to initial low-intensity treatments; see also Siegle, 2020).

Response and engagement are usually early. RCTs for the acute treatment of depression usually last 12-24 weeks. However, a fine-grained examination of the time course of change in most of these RCTs suggests that treatment effects are usually evident very quickly. Meta-analytic reviews of patterns of change in face-to-face psychotherapy trials suggest that large and stable symptom changes, usually around session 5, account for most of the overall change observed (Shalom \& Aderka, 2020). My colleagues and I have reported similar patterns of change in depression and anxiety in our trial of remotely-delivered guided self-help CBT (see Figure 1 panel A; Lorenzo-Luaces, Howard, et al., 2021). Most of the change in pharmacotherapy trials also occurs early in treatment, usually within two weeks, when the separation from placebo is most pronounced (Posternak and Zimmerman, 2005; see also Zilcha-Mano et al., 2017). Data on the neurobiological effects of SSRIs and other medications also suggests that they can produce neurobiological effects (Malhi et al., 2020) immediately, reflecting patterns 
of symptoms improvements. Thus, existing data suggest that large gains are characteristic of active treatments, not placebos (Zilcha-Mano et al., 2017), and predict sustained response (Beard \& Delgadillo, 2019).

The early treatment period is also vital for determining treatment engagement. For example, in a sample of outpatients $(\mathrm{N}=264)$ my colleague and I documented very high rates $(52 \%)$ of dropout in the pre-treatment waiting list period for outpatient psychotherapy (Krendl \& Lorenzo-Luaces, 2021). This pre-treatment dropout accounted for $72.0 \%$ of all dropout that was observed during treatment. Likewise, in our sample of individuals accessing guided self-help CBT for depression and anxiety, in which pre-treatment waiting was relatively homogeneous between individuals and was overall low (i.e., around a week), we also found high rates of pretreatment dropout (43.4\%), which accounted for $79.3 \%$ of all dropout observed during the intervention (see Figure 1 panel B). Others have also observed that pre-treatment and early dropout accounts for the majority of dropout observed during therapy (Bentley et al., 2021). Taken together, these data suggest that the early treatment phase is an important period in which dropout rate is high and in which treatment response may be evident.

Internalizing distress for participants engaging in guided self-help CBT $(N=107)$
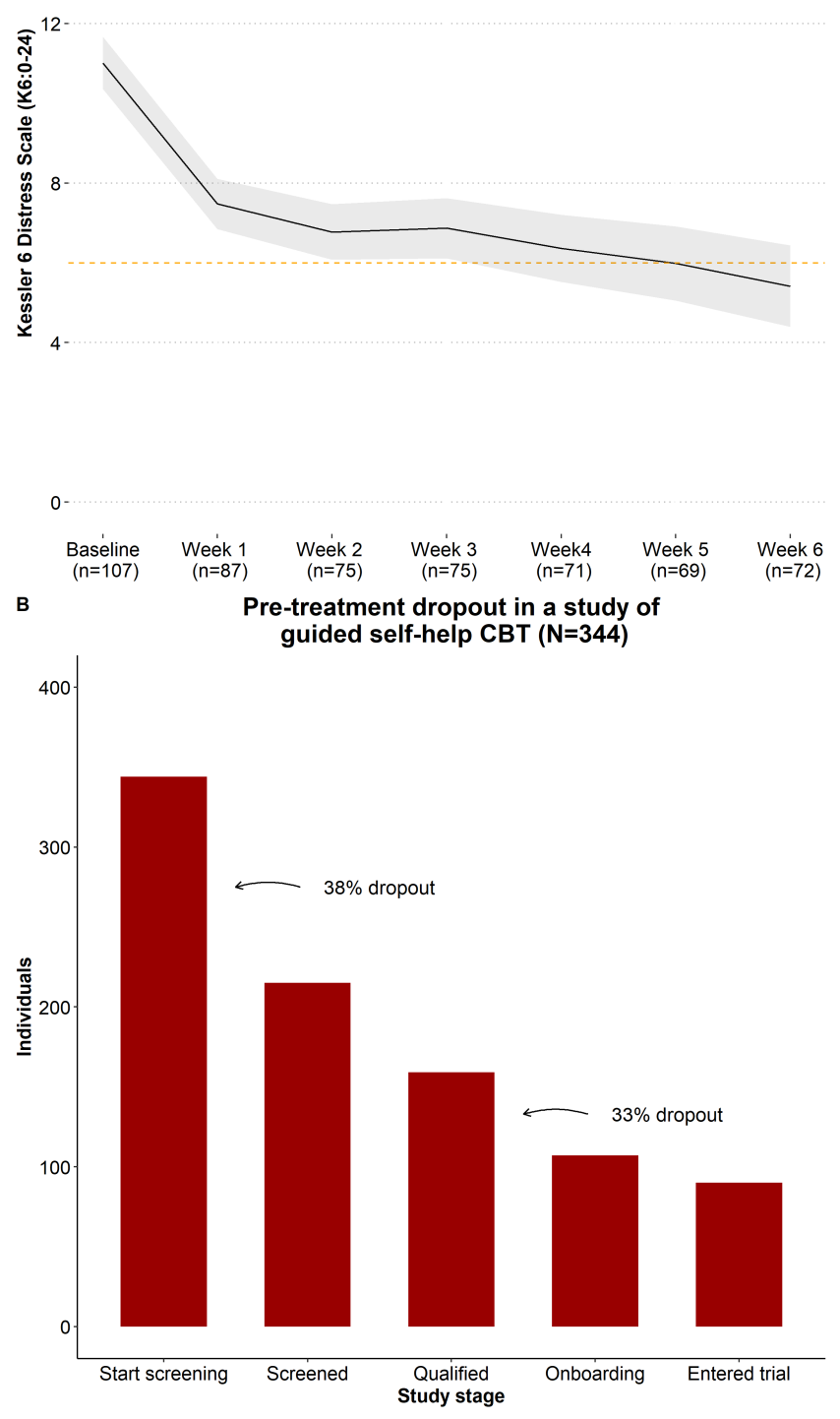

Note. A: $\mathrm{CBT}=$ cognitive-behavioral therapy. $\mathrm{Y}$ axis presented at baseline mean $+/-1 \mathrm{SD}$. Red and yellow doted lines indicate K6 cut-off for severe (13) and mild (5) psychological distress, respectively. B: CBT = cognitive-behavioral therapy. 
Accordingly, in a stepped care treatment program, each step should be of relatively short duration and treatment should begin relatively quickly. The role of "long-term" psychotherapy in treatment resistant depression is less clear (Trivedi et al., 2011) and should be studied further. ${ }^{3}$

Depression as internalizing distress. Evidence supports considering depression on a continuum with sad mood rather than categorically (Ruscio, 2019) ${ }^{4}$. Psychometric (see Ringwald et al., 2021) and behavioral genetic (Pettersson et al., 2016) studies converge on the idea that depressive symptoms co-vary with other symptoms including anxiety, panic, and posttraumatic stress in what has been called an "internalizing" factor of psychopathology (Kotov et al., 2017). Thus, while I have used the term "depression" as a useful category, the arguments presented generally apply to the treatment of symptoms of internalizing distress.

Dimensional and transdiagnostic conceptualizations of psychopathology have existed for over 20 years (see Beutler and Malik, 2002 for examples). Unfortunately, they do not solve many of the problems attributable to the DSM categories like heterogeneity across clinical problems (Haeffel et al., 2021). While there is much excitement about the treatment implications of "transdiagnostic" treatments, studies suggest that existing treatments like CBT are already transdiagnostic, at least to some extent. Exposure to feared stimuli is an effective way of alleviating distress and fear-based symptoms across DSM anxiety disorders (Hofmann \& Smits, 2008; Laska et al., 2014). Emotion regulation experiments suggest that cognitive reappraisal, the analogue to thought restructuring in CBTs (Hofmann \& Asmundson, 2008), is an effective strategy for increasing positive affect and reducing different types of negative affect (Webb et al., 2012). Similarly, meta-analytic reviews suggest that CBTs have effects beyond their primary symptom targets. For example, CBTs for depression are associated with improvements in anxiety (Weitz et al., 2018) and CBTs for anxiety are associated with improvements in depression

\footnotetext{
${ }^{3}$ Some studies suggest the presence of "late responders" in therapies for depression (e.g., Keefe et al., 2019), this pattern of late response is usually associated with negative prognostic factors (e.g., personality pathology) and worse outcomes than early response, and it is unclear to what extent late response is attributable to treatment effects.

${ }^{4}$ This research is not conclusive in the sense that most of it has been conducted mostly with cross-sectional and self-report data so it is possible that different patterns of findings could emerge from analyzing more "objective" markers of psychopathology or from studying the dimensional vs. categorical nature of intensive longitudinal data across individuals.
} 


\footnotetext{
${ }^{5}$ There is some exciting evidence about the possibility that some transdiagnostic treatments target transdiagnostic mechanisms like neuroticism and mindfulness more so than existing treatments (Sauer-Zavala et al., 2021; Woods et al., 2020). Nonetheless, these findings need to be replicated. More importantly, they need to be contextualized with an explanation as to why a treatment would have stronger effects than another in one "key" mechanism but not produce superior outcomes in targeting primary or secondary symptoms (Lorenzo-Luaces et al., 2015; Lorenzo-Luaces et al., 2016).
} 
I have discussed seemingly disparate findings regarding the epidemiology, treatment, and phenomenology of depression. Below are some concrete future directions for depression treatment research, that take into account existing clinical and epidemiological data.

Importance of psychological interventions. If asked to choose between the two, most individuals (69\%, 95\% CI: 0.61 - 0.77; McHugh et al., 2013) prefer psychological interventions to pharmacological ones. Acute treatment with CBT is associated with better depression outcomes than acute and sustained treatment with SSRIs (Furukawa et al., 2021). Moreover, psychological interventions have been linked to improved educational (Baskin et al., 2010; Becker et al., 2014; Kase et al., 2017) and vocational outcomes (e.g., fewer sickness days attributable to mental health; Finnes et al., 2019; Salomonsson, Hedman-Lagerlöf, et al., 2018). Taken together, these data support the need to prioritize the accessibility of psychological interventions. The United Kingdom's Improving Access to Psychological Therapies (IAPT) program, provides an example of a public health program that prioritizes the availability of psychological services (see Wakefield et al., 2021 for a review). It would be wise for federal funding priorities in the U.S. to reflect the importance of psychotherapeutic interventions for addressing the burden of mental health (Teachman et al., 2019). In addition to psychotherapeutic interventions, there are various behavioral or psychosocial interventions that do not require a trained psychotherapist or medical doctor and that may be efficacious in alleviating internalizing disorder symptoms while also affecting social determinants of health including: direct financial support (Haushofer et al., 2020), befriending (Mead et al., 2010), and exercise (Gordon et al., 2018). Further work should clarify the contribution of psychological interventions like CBTs to these other psychosocial interventions effects (Haushofer et al., 2020).

Larger and more representative samples. In the U.S., internet access is nearly ubiquitous (Perrin \& Atske, 2021). There are known disparities in reliable and fast internet access (e.g., it is lower in rural areas; Perrin and Atske, 2021), but accessing mental health providers via telehealth is often easier than in individual face-to-face settings (Kazdin \& Blase, 2011), and both modalities have roughly equal outcomes (Cuijpers et al., 2019). This, coupled with the availability of new technologies (i.e., internet-based CBT) allows recruitment and treatment 
of more representative samples. Indeed, nationwide RCTs with large and fairly representative samples have been shown to be feasible (Arean et al., 2016; Dobias et al., 2021). Even staying within one state (e.g., due to licensing restrictions), opening participant recruitment beyond small clinics increases the representativeness of the sample. More demographically representative samples are needed in treatment research but in order to be able to properly study how the heterogeneity in depression predicts outcomes the clinical entry criteria in RCTs also should also permit recruiting clinically-heterogeneous samples (Lorenzo-Luaces, 2018a). Although the specific operationalizations vary, most RCTs exclude individuals who have histories of substance use, psychosis, or suicidality, or whose symptoms are too mild (Lorenzo-Luaces, Johns, et al., 2018; Lorenzo-Luaces, Zimmerman, et al., 2018). The use of these criteria reduces the number of people who are able to obtain treatment from trials and restricts the utility of potentially predictive baseline information collected by these studies. For example, pooling RCTs of antidepressants vs. placebos can yield very large samples that could be used to study individual differences in treatment response (e.g., Furukawa et al., 2018). However, all (100\%) of these studies exclude patients based on a minimum symptom cut-off, usually excluding patients with mild depression (Zimmerman et al., 2015), which limit their ability to inform the question of whether baseline symptom severity is a useful predictor of differential response to antidepressants vs. placebos (Lorenzo-Luaces, 2018a). Additionally, exclusion criteria sometime have inadvertent effects on the composition of samples (Zimmerman et al., 2017). For example, excluding patients on the basis of substance use risk would mean studies are more likely to exclude men, younger individuals, and Native Americans (Gutkind et al., 2021).

Plurality of treatments. A variety of different levels of care needs to be made available for depression, including low-intensity treatments (e.g., guided CBT self-help) and interventions that extend beyond CBTs and SSRIs (e.g., psychopharmacology, ECT). As well, there needs to be plurality in the biological and psychosocial vulnerabilities that are targeted by treatments. While CBT is a gold standard psychological intervention (Lorenzo-Luaces, 2018b), many patients will not respond to CBT specifically but may respond to psychological interventions that target other mechanisms including positive emotionality (Lopez-Gomez et al., 2019) 
and interpersonal processes (Lopez-Gomez et al., 2019; van Bronswijk et al., 2021).

Dissemination of low-intensity treatments. Ten years ago, Kazdin and Blase (2011) argued that the field needed to move past thinking about individual and face-to-face psychological interventions as the only interventions for mental health problems. They urged for more research on technology (e.g., the internet, apps), other forms of self-help, paraprofessionals, and other media. Much work has documented the efficacy of low-intensity treatments using the internet and digital apps (Cuijpers et al., 2019), but these interventions are not widely used by psychologists (Peipert \& Lorenzo-Luaces, 2021) or the general public. A handful of mental health apps, for example, account for the majority of users of mental health apps (Wasil, Gillespie, et al., 2021; Wasil, Palermo, et al., 2021), and the apps that are most popular are often not the ones using techniques that have a good evidence base (Wasil et al., 2019). Conversely, when researchers find individual self-help materials efficacious, they rarely make these publicly available (Buss et al., 2020). Better dissemination of low-intensity resources is therefore strongly warranted. While there are efforts to disseminate digital interventions (e.g., NIMH RFA-MH-20-510), digital interventions are not the only forms of low-intensity treatments that exist. Individuals may have equally strong (Wasil et al., 2020), or stronger preferences (De Jesús-Romero et al., 2021; Smith et al., 2017), for treatment modalities that are not digital (e.g., books).

The efficacy and scalability of self-help interventions may prompt one to question just how "low," low-intensity interventions can be. Single-session interventions (Schleider \& Weisz, 2018), as their name imply are single session of psychological treatments, usually focused around one mechanism of vulnerability. Single-session interventions appear to be effective when compared to inactive and active controls, though the effect sizes are modest (Schleider \& Weisz, 2018). One advantage of unguided digital single-session interventions is that the intervention can be delivered immediately, reaching many of the individuals who would drop out in the "pre-treatment" period of other modalities.

Integrating treatment steps past low-intensity treatments. There have been several published studies exploring the efficacy of stepped care interventions (see van Straten et al., 
2015, particularly the IMPACT model (Unützer et al., 2002), but these interventions usually do not include relatively "low-intensity" step (e.g., randomization to a single-session interventions; Ho et al., 2016). Instead of continuing to research single administrations of low-intensity treatments while espousing the idea that these are intended as parts of stepped care programs, researchers should actually study integrating low-intensity treatments into stepped care models.

Solomansson et al. (2018) provided a great example of a trial illustrating the value of low-intensity treatments in stepped care. They recruited 396 individuals with internalizing disorders in primary care. In Step I of their RCT, they administered guided self-help with bibliotherapy for a 9-week period to all individuals. Guidance in this study consisted of two 35-45 minute meetings with a psychotherapist who provide education and discussed the use of the book. After the 9-week Step I period, individuals who had not remitted entered Step II and were randomized to either continue guided self-help bibliotherapy for 11 weeks or to complete a course of individual face-to-face CBT over 11 weeks. After Step I of the study, 40\% of patients achieved symptom remission. Of the patients who entered Step II $(n=186)$, remission rates were higher in individual face-to-face CBT (39\%) than in continuation guided self-help (19\%). Several things are notable about that study. First, a sizable proportion of individuals reached symptom remission on guided self-help. Second, as has been hypothesized (Lorenzo-Luaces et al., 2017), reducing the number of individuals who are responders to low-intensity treatment, can reveal large differences between high-and low intensity treatments $(\mathrm{OR}=2.73,95 \% \mathrm{CI}$ : 1.44-5.18) that are usually small and not statistically significant in meta-analyses $(\mathrm{OR}=1.30$, 95\% CI: 0.84-2.06; Cuijpers et al., 2019).

Scalable alternatives to self-report. Just as there probably cannot be an individual CBT provider or psychiatrist for every person who will become depressed (Kazdin \& Blase, 2011), it currently does not seem feasible that every such person could afford or access expensive biological tests that could characterize potential biomarkers of response. Nor, is it clear that collection of such biomarker data would be of value when a large proportion of individuals are known to respond to low-intensity treatments. There is an emerging area of research on digitally and passively collected data that goes beyond self-report to measure individual 
differences (Torous et al., 2017). For example, using data from social media, we and others have characterized aspects of vulnerability to depression including circadian patterns of activity (ten Thij et al., 2020), cognitive distortions (Bathina, Ten Thij, et al., 2021), positive and negative affect levels (Bathina, ten Thij, et al., 2021), and the distribution of social networks (Bollen et al., 2011; see Correia et al., 2020). We are currently investigating the relevance of these measures as potential predictors and mechanisms of engagement and outcomes in transdiagnostic guided self-help (NCT04870099). Others have used smartphone data and meta-data to infer variables such as location, temperature, light exposure and heart rate (e.g., Jacobson and Bhattacharya, 2021), though work on the relevance of these variables as predictors and mechanisms of treatment outcomes is still in its infancy.

Conclusion. Overall, the heterogeneity of internalizing disorders and the plurality of effective interventions argues against any "one-size-fits-all" approach. Proposed solutions to the public health problem burden posed by internalizing disorders, need to account for how common internalizing disorders are, how interventions of different intensity may be required (e.g., watchful waiting vs. electroconvulsive therapy), and how, staying within the same type of treatment modality (e.g., outpatient psychotherapy), treatments that target different vulnerability mechanisms may be required for different individuals (e.g., cognitive vs. interpersonal). Some of the promising areas of innovation that are also scalable, like the use data-driven approaches to predict optimal allocation to different interventions, rely on the collection of larger and more heterogenenous samples than traditionally collected in treatment trials. 


\section{References}

Alegría, M., Chatterji, P., Wells, K., Cao, Z., Chen, C.-n., Takeuchi, D., Jackson, J., \& Meng, X.-L. (2008). Disparity in depression treatment among racial and ethnic minority populations in the United States. Psychiatric Services, 59(11), $1264-1272$.

Angst, J., Paksarian, D., Cui, L., Merikangas, K., Hengartner, M. P., Ajdacic-Gross, V., \& Rössler, W. (2016). The epidemiology of common mental disorders from age 20 to 50: Results from the prospective Zurich Cohort Study. Epidemiology and Psychiatric Sciences, 25(1), 24-32.

Arean, P. A., Hallgren, K. A., Jordan, J. T., Gazzaley, A., Atkins, D. C., Heagerty, P. J., \& Anguera, J. A. (2016). The use and effectiveness of mobile apps for depression: Results from a fully remote clinical trial. Journal of Medical Internet Research, $18(12), \mathrm{e} 330$.

Ashwood, J. S., Mehrotra, A., Cowling, D., \& Uscher-Pines, L. (2017). Direct-to-consumer telehealth may increase access to care but does not decrease spending. Health Affairs, 36(3), 485-491.

Bahji, A., Vazquez, G. H., \& Zarate Jr, C. A. (2021). Comparative efficacy of racemic ketamine and esketamine for depression: A systematic review and meta-analysis. Journal of Affective Disorders, 278, 542-555.

Baskin, T. W., Slaten, C. D., Sorenson, C., Glover-Russell, J., \& Merson, D. N. (2010). Does youth psychotherapy improve academically related outcomes? A meta-analysis. Journal of Counseling Psychology, 57(3), 290.

Bathina, K. C., Ten Thij, M., Lorenzo-Luaces, L., Rutter, L. A., \& Bollen, J. (2021). Individuals with depression express more distorted thinking on social media. Nature Human Behaviour, 5(4), 458-466.

Bathina, K. C., ten Thij, M., Valdez, D., Rutter, L. A., \& Bollen, J. (2021). Declining well-being during the COVID-19 pandemic reveals US social inequities. PLOS ONE, e0254114.

Beard, J. I., \& Delgadillo, J. (2019). Early response to psychological therapy as a predictor of depression and anxiety treatment outcomes: A systematic review and meta-analysis. Depression and Anxiety, 36(9), 866-878.

Becker, K. D., Brandt, N. E., Stephan, S. H., \& Chorpita, B. F. (2014). A review of educational outcomes in the children's mental health treatment literature. Advances in School Mental Health Promotion, 7(1), 5-23.

Bentley, K. H., Cohen, Z. D., Kim, T., Bullis, J. R., Nauphal, M., Cassiello-Robbins, C., Sauer-Zavala, S., Sbi, S., Gallagher, M. W., Farchione, T. J., et al. (2021). The nature, timing, and symptom trajectories of dropout from transdiagnostic and single-diagnosis cognitive-behavioral therapy for anxiety disorders. Behavior Therapy, 52(6), 1364-1376.

Beutler, L. E., \& Malik, M. L. (2002). Rethinking the DSM: A psychological perspective. American Psychological Association.

Bollen, J., Gonçalves, B., Ruan, G., \& Mao, H. (2011). Happiness is assortative in online social networks. Artificial Life, 17(3), $237-251$.

Bright, K. S., Mughal, M. K., Wajid, A., Lane-Smith, M., Murray, L., Roy, N., Van Zanten, S. V., Mcneil, D. A., Stuart, S., \& Kingston, D. (2019). Internet-based interpersonal psychotherapy for stress, anxiety, and depression in prenatal women: Study protocol for a pilot randomized controlled trial. Trials, 20(1), 1-11.

Buss, J., Lorenzo-Luaces, L., Banks, G., Horani, D., Rutter, L., \& Wasil, A. R. (2020). Availability of internet-based cognitive behavioral therapies (iCBTs) for depression: A systematic review. PsyArXiv pre-print submitted for publication: https: //psyarxiv.com/2jwgd/.

Cai, C., Peng, Y., Shen, E., Huang, Q., Chen, Y., Liu, P., Guo, C., Feng, Z., Gao, L., Zhang, X., et al. (2021). A comprehensive analysis of the efficacy and safety of COVID-19 vaccines. Molecular Therapy, 29(9), 2794-2805.

Carvalho, A. F., Berk, M., Hyphantis, T. N., \& McIntyre, R. S. (2014). The integrative management of treatment-resistant depression: A comprehensive review and perspectives. Psychotherapy and Psychosomatics, 83(2), 70-88. 
Cipriani, A., Furukawa, T. A., Salanti, G., Chaimani, A., Atkinson, L. Z., Ogawa, Y., Leucht, S., Ruhe, H. G., Turner, E. H., Higgins, J. P., et al. (2018). Comparative efficacy and acceptability of 21 antidepressant drugs for the acute treatment of adults with major depressive disorder: A systematic review and network meta-analysis. Lancet, 391, 1357-1366.

Correia, R. B., Wood, I. B., Bollen, J., \& Rocha, L. M. (2020). Mining social media data for biomedical signals and health-related behavior. Annual Review of Biomedical Data Science, 3, 433-458.

Cuijpers, P. (2016). The future of psychotherapy research: Stop the waste and focus on issues that matter. Epidemiology and Psychiatric Sciences, 25(4), 291-294.

Cuijpers, P., \& Cristea, I. (2016). How to prove that your therapy is effective, even when it is not: A guideline. Epidemiology and Psychiatric Sciences, 25(5), 428-435.

Cuijpers, P., Cristea, I., Weitz, E., Gentili, C., \& Berking, M. (2016). The effects of cognitive and behavioural therapies for anxiety disorders on depression: A meta-analysis. Psychological Medicine, 46(16), 3451-3462.

Cuijpers, P., Donker, T., van Straten, A., Li, J., \& Andersson, G. (2010). Is guided self-help as effective as face-to-face psychotherapy for depression and anxiety disorders? A systematic review and meta-analysis of comparative outcome studies. Psychological Medicine, 40(12), 1943-1957.

Cuijpers, P., Noma, H., Karyotaki, E., Cipriani, A., \& Furukawa, T. A. (2019). Effectiveness and acceptability of cognitive behavior therapy delivery formats in adults with depression: A network meta-analysis. JAMA Psychiatry, 76(7), 700-707.

Cuthbert, B. N., \& Kozak, M. J. (2013). Constructing constructs for psychopathology: The NIMH research domain criteria RDoC. Journal of Abnormal Psychology, 928-937.

De Jesús-Romero, R., Holder-Dixon, A., \& Lorenzo-Luaces, L. (2021). Reporting and representation of ethnic and racial diversity in randomized controlled trials for internet-based cognitive-behavioral therapy. Manuscript in preparation.

Delgadillo, J., Ali, S., Fleck, K., Agnew, C., Southgate, A., Parkhouse, L., Cohen, Z. D., DeRubeis, R. J., \& Barkham, M. (2021). Stratified care vs stepped care for depression: A cluster randomized clinical trial. JAMA Psychiatry, advanced online publication.

DeRubeis, R. J., Cohen, Z. D., Forand, N. R., Fournier, J. C., Gelfand, L. A., \& Lorenzo-Luaces, L. (2014). The personalized advantage index: Translating research on prediction into individualized treatment recommendations. PLOS ONE, 9(1), e83875.

Dobias, M. L., Schleider, J. L., Jans, L., \& Fox, K. (2021). An online, single-session intervention for adolescent self-injurious thoughts and behaviors: Results from a randomized trial. Behavioural Research and Therapy.

Driessen, E., Cuijpers, P., Hollon, S. D., \& Dekker, J. J. (2010). Does pretreatment severity moderate the efficacy of psychological treatment of adult outpatient depression? A meta-analysis. Journal of Consulting and Clinical Psychology, 78(5), 668680 .

Dunlop, B. W., Kelley, M. E., McGrath, C. L., Craighead, W. E., \& Mayberg, H. S. (2015). Preliminary findings supporting insula metabolic activity as a predictor of outcome to psychotherapy and medication treatments for depression. The Journal of Neuropsychiatry and Clinical Neurosciences, 27(3), 237-239.

Eaton, W. W., Anthony, J. C., Gallo, J., Cai, G., Tien, A., Romanoski, A., Lyketsos, C., \& Chen, L.-S. (1997). Natural history of Diagnostic Interview Schedule/DSM-IV major depression: The Baltimore epidemiologic catchment area follow-up. Archives of General Psychiatry, 54(11), 993-999.

Eaton, W. W., Shao, H., Nestadt, G., Lee, B. H., Bienvenu, O. J., \& Zandi, P. (2008). Population-based study of first onset and chronicity in major depressive disorder. Archives of General Psychiatry, 65(5), 513-520.

Filip, P. V., Cuciureanu, D., Diaconu, L. S., Vladareanu, A. M., \& Pop, C. S. (2018). MALT lymphoma: Epidemiology, clinical diagnosis and treatment. Journal of Medicine and Life, 11(3), 187. 
Finnes, A., Enebrink, P., Ghaderi, A., Dahl, J., Nager, A., \& Öst, L.-G. (2019). Psychological treatments for return to work in individuals on sickness absence due to common mental disorders or musculoskeletal disorders: A systematic review and meta-analysis of randomized-controlled trials. International Archives of Occupational and Environmental Health, 92(3), 273-293.

Fried, E. I., Coomans, F., \& Lorenzo-Luaces, L. (2020). The 341, 737 ways of qualifying for the melancholic specifier. The Lancet Psychiatry, 7(6), 479-480.

Furukawa, T. A., Maruo, K., Noma, H., Tanaka, S., Imai, H., Shinohara, K., Ikeda, K., Yamawaki, S., Levine, S., Goldberg, Y., et al. (2018). Initial severity of major depression and efficacy of new generation antidepressants: Individual participant data meta-analysis. Acta Psychiatrica Scandinavica, 137(6), 450-458.

Furukawa, T. A., Shinohara, K., Sahker, E., Karyotaki, E., Miguel, C., Ciharova, M., Bockting, C. L., Breedvelt, J. J., Tajika, A., Imai, H., et al. (2021). Initial treatment choices to achieve sustained response in major depression: A systematic review and network meta-analysis. World Psychiatry, 20(3), 387-396.

Gordon, B. R., McDowell, C. P., Hallgren, M., Meyer, J. D., Lyons, M., \& Herring, M. P. (2018). Association of efficacy of resistance exercise training with depressive symptoms: Meta-analysis and meta-regression analysis of randomized clinical trials. JAMA Psychiatry, 75(6), 566-576.

Gutkind, S., Fink, D. S., Shmulewitz, D., Stohl, M., \& Hasin, D. (2021). Psychosocial and health problems associated with alcohol use disorder and cannabis use disorder in U.S. adults. Drug and alcohol dependence, 229, 109137.

Haeffel, G. J., Jeronimus, B. F., Kaiser, B. N., Weaver, L. J., Soyster, P. D., Fisher, A. J., Vargas, I., Goodson, J. T., \& Lu, W. (2021). Folk classification and factor rotations: Whales, sharks, and the problems with the hierarchical taxonomy of psychopathology (HiTOP). Clinical Psychological Science, 21677026211002500.

Hammen, C. (2005). Stress and depression. Annual Review of Clinical Psychology, 1, 293-319.

Harris, M. G., Kazdin, A. E., Chiu, W. T., Sampson, N. A., Aguilar-Gaxiola, S., Al-Hamzawi, A., Alonso, J., Altwaijri, Y., Andrade, L. H., Cardoso, G., et al. (2020). Findings from World Mental Health Surveys of the perceived helpfulness of treatment for patients with major depressive disorder. JAMA Psychiatry, 77(8), 830-841.

Hasin, D. S., Sarvet, A. L., Meyers, J. L., Saha, T. D., Ruan, W. J., Stohl, M., \& Grant, B. F. (2018). Epidemiology of adult DSM-5 major depressive disorder and its specifiers in the United States. JAMA Psychiatry, 75(4), 336-346.

Haushofer, J., Mudida, R., \& Shapiro, J. P. (2020). The comparative impact of cash transfers and a psychotherapy program on psychological and economic well-being (tech. rep.). National Bureau of Economic Research.

Ho, F. Y.-Y., Yeung, W.-F., Ng, T. H.-Y., \& Chan, C. S. (2016). The efficacy and cost-effectiveness of stepped care prevention and treatment for depressive and/or anxiety disorders: A systematic review and meta-analysis. Scientific Reports, 6(1), 1-10.

Hofmann, S. G., \& Asmundson, G. J. (2008). Acceptance and mindfulness-based therapy: New wave or old hat? Clinical Psychology Review, 28(1), 1-16.

Hofmann, S. G., \& Smits, J. A. (2008). Cognitive-behavioral therapy for adult anxiety disorders: A meta-analysis of randomized placebo-controlled trials. The Journal of Clinical Psychiatry, 69(4), 621-632.

Jacobson, N. C., \& Bhattacharya, S. (2021). Digital biomarkers of anxiety disorder symptom changes: Personalized deep learning models using smartphone sensors accurately predict anxiety symptoms from ecological momentary assessments. Behaviour Research and Therapy, 104013.

Jorm, A. F., Patten, S. B., Brugha, T. S., \& Mojtabai, R. (2017). Has increased provision of treatment reduced the prevalence of common mental disorders? review of the evidence from four countries. World Psychiatry, 16(1), 90-99.

Kase, C., Hoover, S., Boyd, G., West, K. D., Dubenitz, J., Trivedi, P. A., Peterson, H. J., \& Stein, B. D. (2017). Educational outcomes associated with school behavioral health interventions: A review of the literature. Journal of School Health, $87(7), 554-562$. 
Kazdin, A. E., \& Blase, S. L. (2011). Rebooting psychotherapy research and practice to reduce the burden of mental illness. Perspectives on Psychological Science, 6(1), 21-37.

Keefe, J. R., Solomonov, N., Derubeis, R. J., Phillips, A. C., Busch, F. N., Barber, J. P., Chambless, D. L., \& Milrod, B. L. (2019). Focus is key: Panic-focused interpretations are associated with symptomatic improvement in panic-focused psychodynamic psychotherapy. Psychotherapy Research, 29(8), 1033-1044.

Kennis, M., Gerritsen, L., van Dalen, M., Williams, A., Cuijpers, P., \& Bockting, C. (2020). Prospective biomarkers of major depressive disorder: A systematic review and meta-analysis. Molecular Ppsychiatry, 25(2), 321-338.

Kessler, R. C., Berglund, P., Demler, O., Jin, R., Koretz, D., Merikangas, K. R., Rush, A. J., Walters, E. E., \& Wang, P. S. (2003). The epidemiology of major depressive disorder: Results from the National Comorbidity Survey Replication (NCS-R). JAMA, 289(23), 3095-3105.

Kisely, S., Li, A., Warren, N., \& Siskind, D. (2018). A systematic review and meta-analysis of deep brain stimulation for depression. Depression and Anxiety, 35(5), 468-480.

Kotov, R., Krueger, R. F., Watson, D., Achenbach, T. M., Althoff, R. R., Bagby, R. M., Brown, T. A., Carpenter, W. T., Caspi, A., Clark, L. A., et al. (2017). The hierarchical taxonomy of psychopathology (HiTOP): A dimensional alternative to traditional nosologies. Journal of Abnormal Psychology, 126(4), 454.

Krendl, A. C., \& Lorenzo-Luaces, L. (2021). Identifying peaks in attrition after clients initiate mental health treatment in a university training clinic. Psychological Services, advance online publication.

Laska, K. M., Gurman, A. S., \& Wampold, B. E. (2014). Expanding the lens of evidence-based practice in psychotherapy: A common factors perspective. Psychotherapy, 51(4), 467-481.

Lindegaard, T., Hesslow, T., Nilsson, M., Johansson, R., Carlbring, P., Lilliengren, P., \& Andersson, G. (2020). Internet-based psychodynamic therapy vs cognitive behavioural therapy for social anxiety disorder: A preference study. Internet Interventions, 20, 100316 .

Lopez-Gomez, I., Lorenzo-Luaces, L., Chaves, C., Hervas, G., DeRubeis, R. J., \& Vazquez, C. (2019). Predicting optimal interventions for clinical depression: Moderators of outcomes in a positive psychological intervention vs. cognitive-behavioral therapy. General Hospital Psychiatry, 61, 104-110.

Lorenzo-Luaces, L. (2015). Heterogeneity in the prognosis of major depression: From the common cold to a highly debilitating and recurrent illness. Epidemiology and Psychiatric Sciences, 24(6), 466-472.

Lorenzo-Luaces, L. (2018a). Representing the heterogeneity of depression in treatment research. Acta Psychiatrica Scandinavica, $138(4), 360-361$.

Lorenzo-Luaces, L. (2018b). The evidence for cognitive behavioral therapy (CBT). JAMA, 319(8), 831-832.

Lorenzo-Luaces, L., Buss, J. F., \& Fried, E. I. (2021). Heterogeneity in major depression and its melancholic and atypical specifiers: A secondary analysis of STAR* D. BMC Psychiatry, 21(1), 1-11.

Lorenzo-Luaces, L., DeRubeis, R. J., van Straten, A., \& Tiemens, B. (2017). A prognostic index (PI) as a moderator of outcomes in the treatment of depression: A proof of concept combining multiple variables to inform risk-stratified stepped care models. Journal of Affective Disorders, 213, 78-85.

Lorenzo-Luaces, L., German, R. E., \& DeRubeis, R. J. (2015). It's complicated: The relation between cognitive change procedures, cognitive change, and symptom change in cognitive therapy for depression. Clinical Psychology Review, 41, 3-15.

Lorenzo-Luaces, L., Howard, J., De Jesus-Romero, R., Peipert, A., Lind, C., Botts, K., \& Starvaggi, I. (2021). Acceptability and outcomes of a transdiagnostic guided self-help bibliotherapy for internalizing disordersymptoms in adults: A fully remote nationwide opentrial. PsyArxiv pre-print submitted for publication: https://psyarxiv.com/dkgp6/. 
Lorenzo-Luaces, L., Johns, E., \& Keefe, J. R. (2018). The generalizability of randomized controlled trials of self-guided internetbased cognitive behavioral therapy for depressive symptoms: Systematic review and meta-regression analysis. Journal of Medical Internet Research, 20(11), e10113.

Lorenzo-Luaces, L., Keefe, J. R., \& DeRubeis, R. J. (2016). Cognitive-behavioral therapy: Nature and relation to non-cognitive behavioral therapy. Behavior Therapy, 47(6), 785-803.

Lorenzo-Luaces, L., Lemmens, L. H., Keefe, J. R., Cuijpers, P., \& Bockting, C. L. (2021). The efficacy of cognitive behavioral therapy for emotional disorders. Washington, DC, US: American Psychological Association. Handbook of cognitive behavioral therapy: Overview and approaches. pp. (55-90).

Lorenzo-Luaces, L., Peipert, A., Romero, R. D. J., Rutter, L. A., \& Rodriguez-Quintana, N. (2021). Personalized medicine and cognitive behavioral therapies for depression: Small effects, big problems, and bigger data. International Journal of Cognitive Therapy, 14(1), 59-85.

Lorenzo-Luaces, L., Rodriguez-Quintana, N., Riley, T. N., \& Weisz, J. R. (2020). A placebo prognostic index (PI) as a moderator of outcomes in the treatment of adolescent depression: Could it inform risk-stratification in treatment with cognitivebehavioral therapy, fluoxetine, or their combination? Psychotherapy Research, 1-14.

Lorenzo-Luaces, L., Zimmerman, M., \& Cuijpers, P. (2018). Are studies of psychotherapies for depression more or less generalizable than studies of antidepressants? Journal of Affective Disorders, 234, 8-13.

Luedtke, A., Sadikova, E., \& Kessler, R. C. (2019). Sample size requirements for multivariate models to predict between-patient differences in best treatments of major depressive disorder. Clinical Psychological Science, 7(3), 445-461.

Malhi, G. S., Morris, G., Bell, E., \& Hamilton, A. (2020). A new paradigm for achieving a rapid antidepressant response. Drugs, $80(8), 755-764$.

Mattisson, C., Bogren, M., Horstmann, V., Munk-Jörgensen, P., \& Nettelbladt, P. (2007). The long-term course of depressive disorders in the Lundby study. Psychological Medicine, 37(6), 883-891.

McGrath, C. L., Kelley, M. E., Holtzheimer, P. E., Dunlop, B. W., Craighead, W. E., Franco, A. R., Craddock, R. C., \& Mayberg, H. S. (2013). Toward a neuroimaging treatment selection biomarker for major depressive disorder. JAMA Psychiatry, $70(8), 821-829$.

McHugh, R. K., Whitton, S. W., Peckham, A. D., Welge, J. A., \& Otto, M. W. (2013). Patient preference for psychological vs. pharmacologic treatment of psychiatric disorders: A meta-analytic review. The Journal of Clinical Psychiatry, 74(6), 595-602.

Mead, N., Lester, H., Chew-Graham, C., Gask, L., \& Bower, P. (2010). Effects of befriending on depressive symptoms and distress: Systematic review and meta-analysis. The British Journal of Psychiatry, 196(2), 96-101.

Moffitt, T. E., Caspi, A., Taylor, A., Kokaua, J., Milne, B. J., Polanczyk, G., \& Poulton, R. (2010). How common are common mental disorders? Evidence that lifetime prevalence rates are doubled by prospective versus retrospective ascertainment. Psychological Medicine, 40(6), 899-909.

Mojtabai, R. (2009). Unmet need for treatment of major depression in the United States. Psychiatric Services, 60(3), $297-305$.

Mojtabai, R., Olfson, M., Sampson, N. A., Jin, R., Druss, B., Wang, P. S., Wells, K. B., Pincus, H. A., \& Kessler, R. C. (2011). Barriers to mental health treatment: Results from the National Comorbidity Survey Replication. Psychological Medicine, $41(8), 1751-1761$.

Monroe, S. M., \& Harkness, K. L. (2011). Recurrence in major depression: A conceptual analysis. Psychological Review, 118(4), 655-674.

Morishita, T., Fayad, S. M., Higuchi, M.-a., Nestor, K. A., \& Foote, K. D. (2014). Deep brain stimulation for treatment-resistant depression: Systematic review of clinical outcomes. Neurotherapeutics, 11(3), 475-484. 
Morris, Z. S., Wooding, S., \& Grant, J. (2011). The answer is 17 years, what is the question: Understanding time lags in translational research. Journal of the Royal Society of Medicine, 104(12), 510-520.

Nemeroff, C. B. (2021). Management of major depression-yesterday, today, and tomorrow. Psychiatric News, 56 (11), 130.

Newcomb, M. E., \& Mustanski, B. (2010). Internalized homophobia and internalizing mental health problems: A meta-analytic review. Clinical Psychology Review, 30(8), 1019-1029.

Ormel, J., Hollon, S. D., Kessler, R. C., Cuijpers, P., \& Monroe, S. M. (2021). More treatment but no less depression: The treatment-prevalence paradox. Clinical Psychology Review, 102111.

Pamplin II, J. R., \& Bates, L. M. (2021). Evaluating hypothesized explanations for the black-white depression paradox: A critical review of the extant evidence. Social Science $\mathcal{G}$ Medicine, 114085.

Peipert, A., \& Lorenzo-Luaces, L. (2021). Psychotherapists' experiences with treatment waiting lists and perspectives of low-intensity interventions. Manuscript in preparation.

Pellicane, M. J., \& Ciesla, J. A. (2021). Associations between minority stress, depression, and suicidal ideation and attempts in transgender and gender diverse (TGD) individuals: Systematic review and meta-analysis. Clinical Psychology Review, 102113.

Perrin, A., \& Atske, S. (2021). Internet and technology: Digital divides. Pew Research Center.

Pettersson, E., Larsson, H., \& Lichtenstein, P. (2016). Common psychiatric disorders share the same genetic origin: A multivariate sibling study of the swedish population. Molecular Psychiatry, 21(5), 717-721.

Pigott, H. E. (2011). STAR* D: A tale and trail of bias. Ethical Human Psychology and Psychiatry, 13(1), 6-28.

Pigott, H. E. (2015). The STAR* D trial: It is time to reexamine the clinical beliefs that guide the treatment of major depression. The Canadian Journal of Psychiatry, 60(1), 9-13.

Posternak, M. A., \& Zimmerman, M. (2005). Is there a delay in the antidepressant effect? A meta-analysis. The Journal of Clinical Psychiatry, 66(2), 9889.

Prasad, V., Sri, B. S., \& Gaitonde, R. (2020). Bridging a false dichotomy in the COVID-19 response: A public health approach to the "lockdown" debate. BMJ Global Health, 5(6), e002909.

Press, V. G., Huisingh-Scheetz, M., \& Arora, V. M. (2021). Inequities in technology contribute to disparities in COVID-19 vaccine distribution. JAMA Health Forum, 2(3), e210264-e210264.

Ringwald, W. R., Forbes, M. K., \& Wright, A. G. (2021). Meta-analysis of structural evidence for the hierarchical taxonomy of psychopathology (HiTOP) model. Psychological Medicine, 1-14.

Roberts, B. W., Luo, J., Briley, D. A., Chow, P. I., Su, R., \& Hill, P. L. (2017). A systematic review of personality trait change through intervention. Psychological Bulletin, $143(2), 117$.

Rohde, P., Lewinsohn, P. M., Klein, D. N., Seeley, J. R., \& Gau, J. M. (2013). Key characteristics of major depressive disorder occurring in childhood, adolescence, emerging adulthood, and adulthood. Clinical Psychological Science, 1(1), 41-53.

Rottenberg, J., Devendorf, A. R., Kashdan, T. B., \& Disabato, D. J. (2018). The curious neglect of high functioning after psychopathology: The case of depression. Perspectives on Psychological Science, 13(5), 549-566.

Ruscio, A. M. (2019). Normal versus pathological mood: Implications for diagnosis. Annual Review of Clinical Psychology, 15, 179-205.

Rush, A. J., Trivedi, M. H., Wisniewski, S. R., Nierenberg, A. A., Stewart, J. W., Warden, D., Niederehe, G., Thase, M. E., Lavori, P. W., Lebowitz, B. D., et al. (2006). Acute and longer-term outcomes in depressed outpatients requiring one or several treatment steps: A STAR*D report. American Journal of Psychiatry, 163(11), 1905-1917.

Salomonsson, S., Hedman-Lagerlöf, E., \& Öst, L.-G. (2018). Sickness absence: A systematic review and meta-analysis of psychological treatments for individuals on sick leave due to common mental disorders. Psychological Medicine, 48(12), $1954-1965$. 
Salomonsson, S., Santoft, F., Lindsäter, E., Ejeby, K., Ljótsson, B., Öst, L.-G., Ingvar, M., Lekander, M., \& Hedman-Lagerlöf, E. (2018). Stepped care in primary care-guided self-help and face-to-face cognitive behavioural therapy for common mental disorders: A randomized controlled trial. Psychological Medicine, 48(10), 1644-1654.

Sauer-Zavala, S., Fournier, J. C., Steele, S. J., Woods, B. K., Wang, M., Farchione, T. J., \& Barlow, D. H. (2021). Does the unified protocol really change neuroticism? Results from a randomized trial. Psychological Medicine, 51(14), 2378-2387.

Schleider, J. L., \& Weisz, J. (2018). A single-session growth mindset intervention for adolescent anxiety and depression: 9-month outcomes of a randomized trial. Journal of Child Psychology and Psychiatry, 59(2), 160-170.

Schleider, J. L., \& Weisz, J. R. (2017). Can less be more? The promise (and perils) of single-session youth mental health interventions. The Behavior Therapist, 40(7), 256-261.

Shalom, J. G., \& Aderka, I. M. (2020). A meta-analysis of sudden gains in psychotherapy: Outcome and moderators. Clinical Psychology Review, 76, 101827.

Simon, G. E., \& Perlis, R. H. (2010). Personalized medicine for depression: Can we match patients with treatments? American Journal of Psychiatry, 167(12), 1445-1455.

Smith, J., Newby, J. M., Burston, N., Murphy, M. J., Michael, S., Mackenzie, A., Kiln, F., Loughnan, S. A., O’Moore, K. A., Allard, B. J., et al. (2017). Help from home for depression: A randomised controlled trial comparing internet-delivered cognitive behaviour therapy with bibliotherapy for depression. Internet Interventions, 9, 25-37.

Spijker, J., De Graaf, R., Bijl, R. V., Beekman, A. T., Ormel, J., \& Nolen, W. A. (2002). Duration of major depressive episodes in the general population: Results from the Netherlands Mental Health Survey and Incidence Study (NEMESIS). The British Journal of Psychiatry, 181 (3), 208-213.

Spinhoven, P., Klein, N., Kennis, M., Cramer, A. O., Siegle, G., Cuijpers, P., Ormel, J., Hollon, S. D., \& Bockting, C. L. (2018). The effects of cognitive-behavior therapy for depression on repetitive negative thinking: A meta-analysis. Behaviour Research and Therapy, 106, 71-85.

Steinert, C., Hofmann, M., Kruse, J., \& Leichsenring, F. (2014). The prospective long-term course of adult depression in general practice and the community: A systematic literature review. Journal of Affective Disorders, 152, 65-75.

Takayanagi, Y., Spira, A. P., Roth, K. B., Gallo, J. J., Eaton, W. W., \& Mojtabai, R. (2014). Accuracy of reports of lifetime mental and physical disorders: Results from the Baltimore Epidemiological Catchment Area study. JAMA Psychiatry, 71(3), $273-280$.

Teachman, B. A., McKay, D., Barch, D. M., Prinstein, M. J., Hollon, S. D., \& Chambless, D. L. (2019). How psychosocial research can help the [national institute of mental health] achieve its grand challenge to reduce the burden of mental illnesses and psychological disorders. American Psychologist, 74(4), 415.

ten Thij, M., van de Leemput, I., Bathina, K., Rutter, L., Lorenzo-Luaces, L., Scheffer, M., \& Bollen, J. (2020). Depression alters circadian pattern of online activity. Scientific Reports, 17272.

The UK ECT Review Group. (2003). Efficacy and safety of electroconvulsive therapy in depressive disorders: A systematic review and meta-analysis. The Lancet, 361 (9360), 799-808.

Torous, J., Onnela, J., \& Keshavan, M. (2017). New dimensions and new tools to realize the potential of RDoC: Digital phenotyping via smartphones and connected devices. Translational Psychiatry, 7(3), e1053-e1053.

Trivedi, R. B., Nieuwsma, J. A., \& Williams, J. W. (2011). Examination of the utility of psychotherapy for patients with treatment resistant depression: A systematic review. Journal of General Internal Medicine, 26 (6), 643-650.

Turner, E. H. (2013). Publication bias, with a focus on psychiatry: Causes and solutions. CNS drugs, 27(6), 457-468.

Unützer, J., Katon, W., Callahan, C. M., Williams Jr, J. W., Hunkeler, E., Harpole, L., Hoffing, M., Della Penna, R. D., Noël, P. H., Lin, E. H., et al. (2002). Collaborative care management of late-life depression in the primary care setting: A randomized controlled trial. JAMA, 288(22), 2836-2845. 
van Bronswijk, S. C., Bruijniks, S. J., Lorenzo-Luaces, L., Derubeis, R. J., Lemmens, L. H., Peeters, F. P., \& Huibers, M. J. (2021). Cross-trial prediction in psychotherapy: External validation of the Personalized Advantage Index using machine learning in two Dutch randomized trials comparing CBT versus IPT for depression. Psychotherapy Research, 31(1), 78-91.

van Bronswijk, S. C., Moopen, N., Beijers, L., Ruhe, H. G., \& Peeters, F. (2019). Effectiveness of psychotherapy for treatmentresistant depression: A meta-analysis and meta-regression. Psychological Medicine, 49(3), 366-379.

van Straten, A., Hill, J., Richards, D. A., \& Cuijpers, P. (2015). Stepped care treatment delivery for depression: A systematic review and meta-analysis. Psychological Medicine, 45(2), 231-246.

Wakefield, S., Kellett, S., Simmonds-Buckley, M., Stockton, D., Bradbury, A., \& Delgadillo, J. (2021). Improving Access to Psychological Therapies (IAPT) in the United Kingdom: A systematic review and meta-analysis of 10-years of practice-based evidence. British Journal of Clinical Psychology, 60(1), 1-37.

Wasil, A. R., Gillespie, S., Schell, T., Lorenzo-Luaces, L., \& DeRubeis, R. J. (2021). Estimating the real-world usage of mobile apps for mental health: Development and application of two novel metrics. World Psychiatry, 20(1), 137.

Wasil, A. R., Malhotra, T., Tuteja, N., Nandakumar, N., Pandole, L., DeRubeis, R., Lorenzo-Luaces, L., Naslund, J., \& Bhatia, A. (2020). Preferences toward digital and non-digital mental health treatment delivery formats: A survey of Indian college students. PsyArXiv pre-print submitted for publication: https://psyarxiv.com/q8krz/.

Wasil, A. R., Palermo, E. H., Lorenzo-Luaces, L., \& DeRubeis, R. J. (2021). Is there an app for that? a review of popular apps for depression, anxiety, and well-being. Cognitive and Behavioral Practice.

Wasil, A. R., Venturo-Conerly, K. E., Shingleton, R. M., \& Weisz, J. R. (2019). A review of popular smartphone apps for depression and anxiety: Assessing the inclusion of evidence-based content. Behaviour Research and Therapy, 123, 103498.

Webb, T. L., Miles, E., \& Sheeran, P. (2012). Dealing with feeling: A meta-analysis of the effectiveness of strategies derived from the process model of emotion regulation. Psychological Bulletin, 138(4), 775-808.

Weitz, E., Kleiboer, A., van Straten, A., \& Cuijpers, P. (2018). The effects of psychotherapy for depression on anxiety symptoms: A meta-analysis. Psychological Medicine, 48(13), 2140-2152.

Wells, J. E., \& Horwood, L. J. (2004). How accurate is recall of key symptoms of depression? A comparison of recall and longitudinal reports. Psychological Medicine, 34(6), 1001-1011.

Whiteford, H. A., Degenhardt, L., Rehm, J., Baxter, A. J., Ferrari, A. J., Erskine, H. E., Charlson, F. J., Norman, R. E., Flaxman, A. D., Johns, N., et al. (2013). Global burden of disease attributable to mental and substance use disorders: Findings from the Global Burden of Disease Study 2010. The Lancet, 382(9904), 1575-1586.

Wilhelm, K., Mitchell, P. B., Niven, H., Finch, A., Wedgwood, L., Scimone, A., Blair, I. P., Parker, G., \& Schofield, P. R. (2006). Life events, first depression onset and the serotonin transporter gene. The British Journal of Psychiatry, 188(3), 210-215.

Woods, B. K., Sauer-Zavala, S., Farchione, T. J., \& Barlow, D. H. (2020). Isolating the effects of mindfulness training across anxiety disorder diagnoses in the unified protocol. Behavior Therapy, 51(6), 972-983.

Zilcha-Mano, S., Roose, S. P., Brown, P. J., \& Rutherford, B. R. (2017). Early symptom trajectories as predictors of treatment outcome for citalopram versus placebo. The American Journal of Geriatric Psychiatry, 25(6), 654-661.

Zimmerman, M. (2015). Does the adequacy of clinicians diagnostic practice in routine clinical settings matter? The Journal of Clinical Psychiatry, 76(7), e888-e890.

Zimmerman, M., Clark, H. L., Multach, M. D., Walsh, E., Rosenstein, L. K., \& Gazarian, D. (2015). Have treatment studies of depression become even less generalizable? A review of the inclusion and exclusion criteria used in placebo-controlled antidepressant efficacy trials published during the past 20 years. Mayo Clinic Proceedings, 90(9), 1180-1186.

Zimmerman, M., Walsh, E., Chelminski, I., \& Dalrymple, K. (2017). Has the symptom severity inclusion requirement narrowed the definition of major depressive disorder in antidepressant efficacy trials? Journal of Affective Disorders, 211, 60-64. 